\title{
Quantum-confined direct-gap transitions in tensile-strained Ge/SiGe multiple quantum wells
}

\author{
Lee Carroll, ${ }^{1, a)}$ Florian Imbert, ${ }^{1}$ Hans Sigg, ${ }^{1}$ Martin Süess, ${ }^{2}$ Elisabeth Müller, ${ }^{2}$ \\ Michele Virgilio, ${ }^{3,4}$ Giovanni Pizzi, ${ }^{3,5}$ Peggy Rossbach, ${ }^{6}$ Daniel Chrastina, ${ }^{7}$ \\ and Giovanni Isella ${ }^{7}$ \\ ${ }^{1}$ Laboratory for Micro- and Nanotechnology, Paul Scherrer Institut, CH-5232, Switzerland \\ ${ }^{2}$ Electron Microscopy ETH Zurich (EMEZ), ETH-Zurich, CH-8093, Switzerland \\ ${ }^{3}$ NEST, Istituto di Nanoscienze, CNR-Nano, I-56127 Pisa, Italy \\ ${ }^{4}$ Dipartimento di Fisica "E. Fermi”, Università di Pisa, I-56127 Pisa, Italy \\ ${ }^{5}$ Scuola Normale Superiore, Piazza dei Cavalieri 7, I-56126 Pisa, Italy \\ ${ }^{6}$ EMPA-Duebendorf, $\mathrm{CH}-8600$ Dübendorf, Switzerland \\ ${ }^{7}$ L-NESS, Dip. di Fisica del Politecnico di Milano, Polo di Como, via Anzani 42, I-22100 Como, Italy
}

(Received 14 February 2011; accepted 3 June 2011; published online 22 July 2011)

\begin{abstract}
Tensile-strained $\mathrm{Ge} / \mathrm{Si}_{1-\mathrm{x}} \mathrm{Ge}_{\mathrm{x}}(\mathrm{x}=0.87)$ multiple quantum wells (MQWs) on a Ge-on-Si virtual substrate are investigated with Brewster transmission and photo-reflectance, to identify quantumconfined direct-gap transitions and their light/heavy-hole splitting. Strain is deduced from optical splitting and $\mathrm{x}$-ray diffraction measurements. As-prepared MQWs have an exciton at $\approx 820 \mathrm{meV}$, close to the $810 \mathrm{meV}$ edge of the telecommunication $\mathrm{C}$-band. The effect of rapid thermal annealing, to redshift this feature into the C-band via increased strain, is investigated and interpreted with a tight-binding model. Annealing is observed to red-shift bulk absorption, but MQW transitions experience a net blue-shift due to interdiffusion. (C) 2011 American Institute of Physics. [doi:10.1063/1.3606383]
\end{abstract}

To integrate Ge/SiGe multiple quantum wells (MQWs) into a complementary metal-oxide semiconductor compatible telecom platform, tensile strain is needed to lower the direct-gap (DG) transitions in the quantum wells (QWs) towards the telecommunication windows. Of particular interest are suitably strained MQW detectors and modulators ${ }^{1}$ where the absorption onset and sharp exciton resonance overlap with the 1.530-1.565 $\mu \mathrm{m}$ (810-792 meV) C-band window. Furthermore, optically pumped lasing has recently been reported from n-type doped, tensile strained Ge-on-Si layers. $^{2}$ For this bulk Ge-on-Si laser system, the tensile-strain reduces the direct-indirect band separation (between the $\Gamma$ and $\mathrm{L}$ conduction band minima), increasing the population of electrons in the $\Gamma$-valley and promoting DG emission. While quantum-confined DG transitions, ${ }^{3}$ Stark-shifts, ${ }^{4}$ and transient gain ${ }^{5}$ have already been observed in compressively-strained Ge/SiGe MQWs, results from tensile-strained $\mathrm{Ge} / \mathrm{SiGe}$ MQWs have only recently been reported. Y Chen et al. have shown that tensile-strained Ge/SiGe MQWs on a Ge-on-Si virtual substrate (VS) can be prepared, ${ }^{6}$ but present only $100 \mathrm{meV}$-broad room-temperature (RT) photoluminescence (PL) peaks as evidence of quantum-confined DG transitions. In this letter, we use transmission and photoreflectance spectroscopy to provide definitive evidence of quantum-confined DG transitions in tensile-strained $\mathrm{Ge} / \mathrm{SiGe}$ MQWs. Increased sample strain after post-growth rapid thermal annealing (RTA) is investigated, and the effect on the DG transitions measured and modelled using a tight-binding (TB) approach. ${ }^{7}$

The 10-period Ge/SiGe MQW heterostructures and $2 \mu \mathrm{m}$ Ge-on-Si VS were grown using low-energy plasma-enhanced chemical vapor deposition (LEPECVD). ${ }^{8}$ The Ge-on-Si VS was grown at $500{ }^{\circ} \mathrm{C}$ on a Boron-doped (1-10 $\left.\Omega \mathrm{cm}\right) \mathrm{Si}$-sub-

${ }^{a)}$ Electronic mail: lee.b.carroll@gmail.com. strate, at a rate of $4.2 \mathrm{~nm} \mathrm{~s}^{-1}$, and then annealed 6 times between $600{ }^{\circ} \mathrm{C}$ and $820{ }^{\circ} \mathrm{C}$ with a ramp rate of $2{ }^{\circ} \mathrm{C} \mathrm{s}^{-1}$. Photodiodes fabricated from similar material demonstrate good responsivity and low dark current, indicating a low threading dislocation density and background doping ${ }^{9}$ (estimated $<1 \times 10^{17} \mathrm{~cm}^{-3}$ ). The MQW structures were grown on the VS at $520^{\circ} \mathrm{C}$ with a nominal rate of 0.26 and $0.29 \mathrm{~nm} \mathrm{~s}^{-1}$ for the QWs and barriers, respectively. X-ray diffraction (XRD) measurements give nominal barrier and QW widths of $8.8 \mathrm{~nm}$ and $17.6 \mathrm{~nm}$, respectively. The barrier composition is determined as $\mathrm{Si}_{1-x} \mathrm{Ge}_{x}(x=0.87)$, and the QWs pure $\mathrm{Ge}(>99 \%)$. Secondary ion mass spectroscopy was used to verify that the Ge-layer in the VS was Si-free to $<0.1 \%$. Brewster transmission and photo-reflection measurements were carried-out on $7 \mathrm{~mm} \times 7 \mathrm{~mm}$ "chips" diced from the sample wafer, and chemical-mechanically polished on the back face. A series of post-growth RTA samples, for enhanced tensile-strain, was prepared between $650{ }^{\circ} \mathrm{C}$ and $850{ }^{\circ} \mathrm{C}$ in an inert-atmosphere oven. The sample chips were held at their maximum temperature for $30 \mathrm{~s}(5 \mathrm{~s}$ and $1 \mathrm{~s}$ for "flash-annealed" samples) and cooled at $28{ }^{\circ} \mathrm{C} / \mathrm{s}$ (from $850-600{ }^{\circ} \mathrm{C}$ ), limited by the thermal inertia of the oven. During annealing, the chips were protected with a $200 \mathrm{~nm}$ PECVD silicon oxide layer.

Figure 1(a) shows the transmission-spectrum (and firstderivative) of the as-prepared MQW sample, along with the first-derivative spectra of a series 30 s RTA samples. The sharp features below $800 \mathrm{meV}$ are due to the light-hole to the conduction band $\Gamma$ point $(\mathrm{LH}-\mathrm{c} \Gamma)$, and heavy-hole to conduction band $\Gamma$ point $(\mathrm{HH}-\mathrm{c} \Gamma)$, absorption edges of the bulk Ge-on-Si VS. For moderate in-plane strain, this VS LH$\mathrm{HH}$ splitting $(\Delta)$ is linearly related to the biaxial tensilestrain $\left(\varepsilon_{/ /}\right)$by $\Delta=(6700 \pm 50) \mathrm{meV} \times \varepsilon_{/ / /}{ }^{10}$ Without postgrowth RTA, the VS biaxial tensile-strain (as deduced from transmission) is $0.17 \%$ from the sample growth conditions. This was independently verified using XRD rocking-curve 

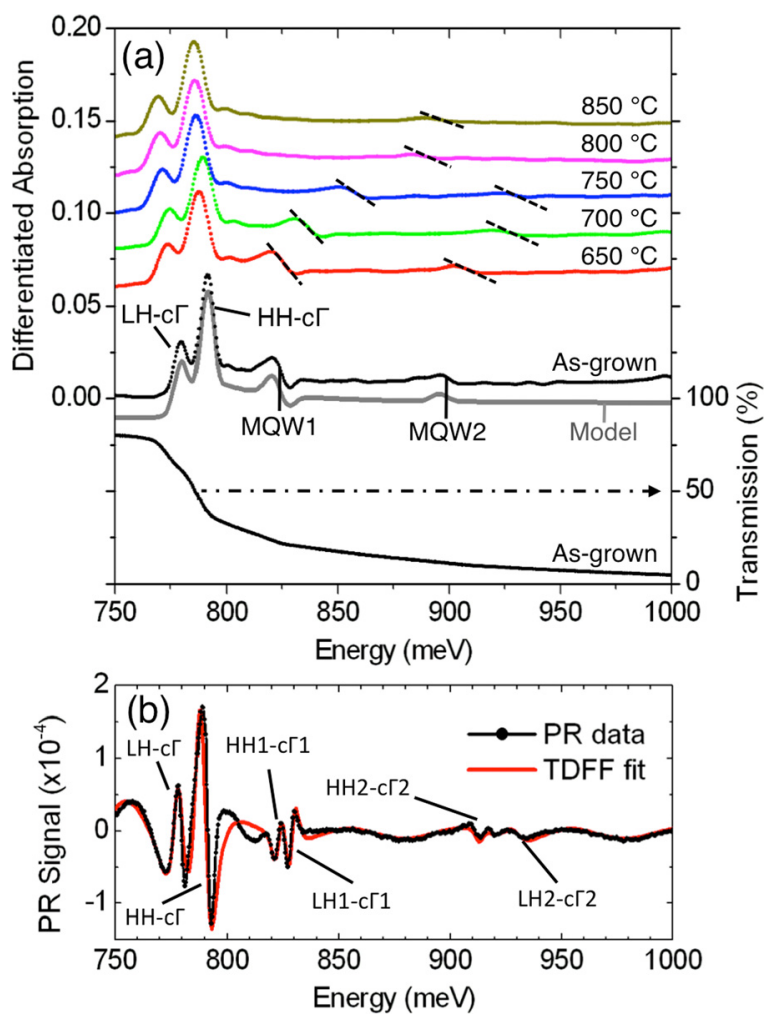

FIG. 1. (Color online) (a) Brewster-geometry transmission spectrum of the as-prepared sample, and the first-derivative spectra on the RTA series. The line-shape is fitted to a qualitative model to extract the MQW1 and MQW2 transition energies. (b) PR-spectrum of the as-prepared sample, clearly resolving individual light/heavy-hole transitions in MQW1 and MQW2. The PRspectrum is fit with a TDFF model to extract the $\mathrm{LH} / \mathrm{HH}$ transition energies.

measurements $\left(\varepsilon_{/ /}=0.16 \pm 0.01 \%\right)$. As they are grown coherently on the VS (verified by XRD reciprocal space map measurements) the Ge/SiGe MQWs also have this $0.17 \%$ strain. Two quantum-confined DG transitions can be resolved in the first-derivative spectra, and using the $\mathrm{TB}$ model they are attributed to the superposition of LH1/HH1с $\Gamma 1$ transitions, termed "MQW1", at $824 \pm 1 \mathrm{meV}$ and to the superposition of $\mathrm{LH} 2 / \mathrm{HH} 2-\mathrm{c} \Gamma 2$ transitions, termed "MQW2", at $895 \pm 3 \mathrm{meV}$, with a dominant contribution from the LH transitions. In fitting the line-shape of the firstderivative spectra, the under-shoot of MQW1 is reproduced by including an exciton feature (in addition to the expected 2D band-to-band transition). Our purely qualitative model uses erf-functions for the 2D band-to-band transitions and a Gaussian peak for the exciton feature.

Photo-reflectance (PR) spectroscopy $^{11}$ was performed using light from a filament lamp, transmitted through a grating spectrometer and focussed to a $750 \mu \mathrm{m}$ spot is used as the probe, with a $50 \mathrm{~mW}, 532 \mathrm{~nm} \mathrm{CW}$ laser for excitation. As shown in Figure 1(b), the PR spectrum of the as-prepared sample resolves the LH-HH splitting of the quantum-confined MQW1 and MQW2 transitions (in addition to the bulk VS splitting). After accounting for the thin-film interference of the Ge-on-Si VS, the PR spectrum can be fitted using the standard third-derivative functional form (TDFF) model, ${ }^{12}$ with exciton, 3D band-to-band, and 2D band-to-band critical points. An exciton feature is needed to properly fit the MQW1 transition. The transmission spectrum of the as-prepared sample gives the MQW1 transition at $824 \pm 1 \mathrm{meV}$, and PR of the same material resolves the $\mathrm{HH} 1-\mathrm{c} \Gamma 1$ transition at $819 \pm 1 \mathrm{meV}$. This indicates that the strained $\mathrm{Ge} / \mathrm{SiGe} \mathrm{MQW}$ s have an exciton feature just 9-14 meV from the infrared telecommunication C-band (Erbium window: $810-792 \mathrm{meV}$ ). An exciton feature so close to the C-band is already of technological interest, because the absorption band can be fully shifted into the band using moderate Stark-effect tuning fields. ${ }^{13}$ The possibility of red-shifting this exciton feature further into the C-band motivated the investigation of the RTA samples.

As expected, RTA increases the strain of the MQWs from $0.17 \%$ to $0.24 \%$, see Figure 2(a), and red-shifts the Geon-Si VS absorption edges. However, both MQW1 and MQW2 transitions experience a blue-shift, as shown in Fig. 2(b), even for the lowest RTA of $650{ }^{\circ} \mathrm{C}$. In the RTA samples, MQW1 and MQW2 can be resolved up to $750{ }^{\circ} \mathrm{C}$ but after $800{ }^{\circ} \mathrm{C}$, only a single feature at $\approx 895 \mathrm{meV}$ in transmission is observed. This is coincides closely with the 902 meV DG of the strained $\mathrm{Si}_{1-x} \mathrm{Ge}_{x}(x=0.96)$ alloy given by the stochiometric mix of QWs and barriers. The net blue-shift of MQW1 away from the C-band is explained by annealinginduced inter-diffusion between the barriers and QWs. A numerical model is constructed, starting with the as-prepared profile (assuming sharp interfaces, periodic boundary conditions, fixed nominal barrier composition and dimensions).

For the annealed samples, alloy inter-diffusion is calculated using the temperature dependent linear diffusion-constant values extrapolated from (i) the literature, ${ }^{14}$ and (ii) from our own XRD determination. As per the XRD data, we assume coherent growth of the MQWs without relaxation of the in-plane tensile-strain. We assume a constant in-plane lattice parameter for each sample, deduced from the VS LH-HH splitting. The $z$-dependent lattice constant in the growth direction is evaluated by means of macroscopic elastic theory, where the Poisson ratio is calculated using (i) the deduced inter-diffusion profile, (ii) the relaxed lattice parameters and (iii) elastic constants of the SiGe, based on a linear interpolation of the pure $\mathrm{Si}$ and $\mathrm{Ge}$ values. The generated layer profiles are then used to calculate the relevant inter-band electronic states by means of a TB code, where the measured Ge concentrations and layer thicknesses are used as input parameters. ${ }^{7}$ In order to better reproduce the MQW electronic states throughout the whole Brillouin zone (BZ), our TB parameterization is obtained semi-empirically to reproduce the overall valence and conduction band dispersion and deformation potentials at the relevant bands edges. ${ }^{3,15,16}$ The calculated transition energies are given in Fig. 2(c), with both the transition energies and the general trend of a blue-shift in MQW1 and MQW2 being reproduced. The measured onset of inter-diffusion (taken as the first net blue-shift of the transitions) occurs at $650{ }^{\circ} \mathrm{C}$, while the model based on literature values predicts $700{ }^{\circ} \mathrm{C}$. Careful calibration of the RTA oven (melting of Al and Ge test samples) confirmed the accuracy of our temperature-readings. The strongerthan-expected diffusion of the $800^{\circ} \mathrm{C}, 750{ }^{\circ} \mathrm{C}$ and $650{ }^{\circ} \mathrm{C} 30 \mathrm{~s}$ annealed samples was verified by XRD analysis of the first super-lattice (SL) satellite peak. ${ }^{17}$ Our SL signal reduction is found to be a factor of 5.5 times stronger than the existing values, equivalent to a $50 \mathrm{~K}$ temperature-shift. It should be noted that reported values of the linear diffusion constant for $\mathrm{Ge} /$ SiGe MQWs can vary by $50 \mathrm{~K}$ (see Refs. 14,18 ). The model based on our own XRD-determined diffusion constant gives 

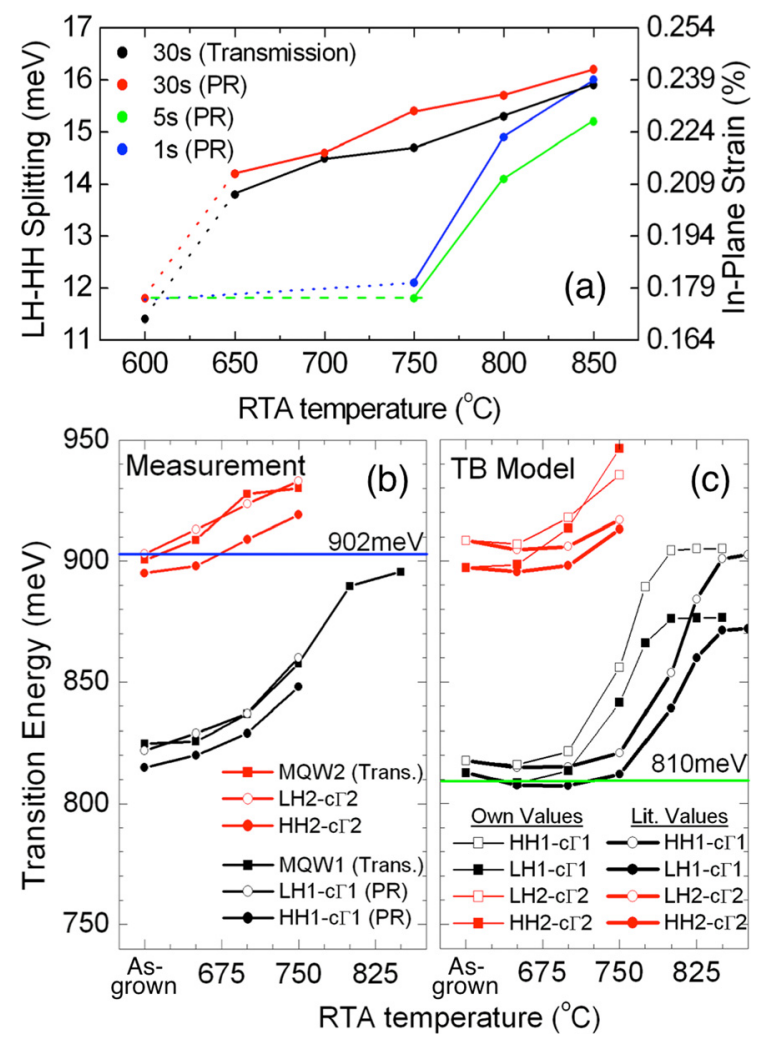

FIG. 2. (Color online) (a) LH-HH splitting from transmission and PR data, and the corresponding biaxial strain of the VS, as a function of RTA time and temperature. (b) Transmission and PR determinations of MQW1 (LH1/ $\mathrm{HH} 1-\mathrm{c} \Gamma 1)$ and MQW2 (LH2/HH2-cГ2) transition energies, as a function of 30 s RTA. (c) TB calculations of the LH1/HH1-c 1 and $\mathrm{LH} 2 / \mathrm{HH} 2-\mathrm{c} \Gamma 2$ transitions, taking the linear diffusion-constant from (i) the literature and (ii) our own XRD determination.

improved agreement, but still predicts only a very modest redshift of MQW1, which could easily be offset by the natural variation in QW thickness in different "chips". In an effort to decouple the role of strain and inter-diffusion, some chips were "flash-annealed" to their maximum temperature for just $1 \mathrm{~s}$ or $5 \mathrm{~s}$. However, as shown in Fig. 2(a), flash-annealing to below $800^{\circ} \mathrm{C}$ does not increase the tensile strain, while annealing for even $1 \mathrm{~s}$ above $800^{\circ} \mathrm{C}$ removes all traces of MQW features from the PR spectra.

The TB calculations in Fig. 2(c) only include the difference between energy states at the $\Gamma$-point of the BZ for MQW1, or the BZ boundary along the growth direction for MQW2. Accounting for the $k_{\mathrm{z}}$ band dispersion could bring the model and the experimental data into even better agreement. The band dispersion calculations, using our XRDdetermined diffusion constant, are used to estimate the annealing-induced transition-broadening, due to joint density of states dispersion along the growth direction. This dispersion is mainly due to coupling of valence states confined in neighbouring QWs and increases for highly inter-diffused QW profiles. Assuming negligible dependence of the dipole matrix elements on $k_{\mathrm{z}}$, the strength of the transmission features are inversely proportional to such broadening. This is clearly demonstrated in Fig. 3, where the measured MQW1

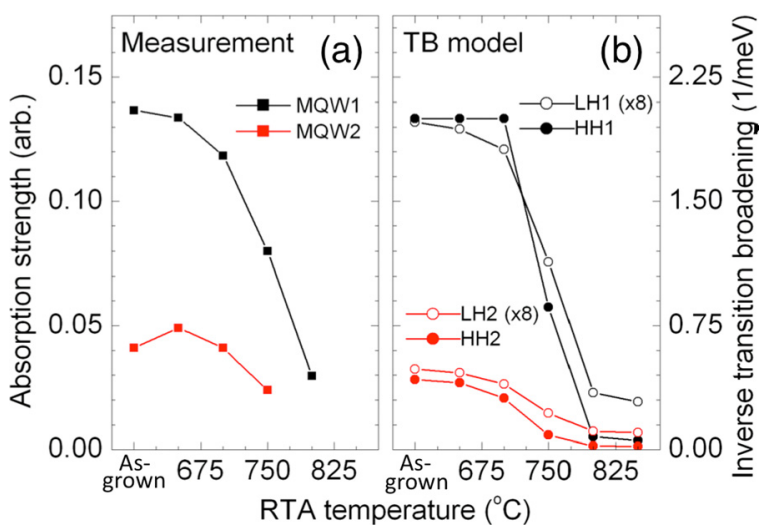

FIG. 3. (Color online) (a) Absorption strength of the MQW1 and MQW2 transitions, as determined from the transmission spectra. (b) The inverse transition broadening of the $\mathrm{LH} 1 / \mathrm{HH} 1-\mathrm{c} \Gamma 1$ and $\mathrm{LH} 2 / \mathrm{HH} 2-\mathrm{c} \Gamma 2$ transitions, as calculated from the TB model taking the linear diffusion-constant from our own XRD determination.

and MQW2 feature-strength and calculated inverse-broadening differ only by a scaling factor.

Finally, transmission electron microscopy (TEM) crosssection images of the as-prepared and $30 \mathrm{~s} 750{ }^{\circ} \mathrm{C}$ annealed samples were compared. The concentration of defects at the interface between the VS and MQWs is significantly higher for the case of the $750{ }^{\circ} \mathrm{C}$ sample. We speculate that these defects act to promote the observed inter-diffusion between the wells and barriers at relatively low temperatures.

In summary, quantum-confined DG transitions have been clearly identified in $0.17-0.24 \%$ tensile-strained $\mathrm{Ge} / \mathrm{SiGe}$ MQWs. The as-prepared MQWs have an exciton feature just 9-14 meV from the C-band edge. TB calculations are used to identify the different $\mathrm{LH} / \mathrm{HH}$ transitions. RTA of the samples increases their strain, red-shifting the VS absorption edges, but the quantum-confined transitions experience a net blue-shift, due to strong inter-diffusion. In future, it is hoped that LEPECVD growth of $\mathrm{Ge} / \mathrm{SiGe} \mathrm{MQWs}$ directly onto $850^{\circ} \mathrm{C}$ preannealed Ge VS will offer the strain needed for $\mathrm{LH} 1-\mathrm{c} \Gamma 1$ to enter the C-band, and bypass inter-diffusion effects entirely.

Part of this work is supported by the Swiss National Science Foundation (SNF\#130181). We thank Stefan Stutz and Anja Weber for sample preparation.

${ }^{1}$ Y-H. Kuo et al., Nature (London) 437, 1334 (2005).

${ }^{2}$ J. Liu et al., Opt. Lett., 35, 679 (2010).

${ }^{3}$ M. Virgilio et al., Phys. Rev. B 79, 075323 (2009).

${ }^{4}$ S. Tsujino et al., Appl. Phys. Lett. 89, 262119 (2006).

${ }^{5}$ C. Lange et al., Phys. Rev. B 79, 201306R (2009).

${ }^{6}$ Y. Chen, C. Li, H. Lai, and S. Chen, Nanotechnology 21115207 (2010).

${ }^{7}$ G. Pizzi, M. Virgilio, and G. Grosso, Nanotechnology 21, 055202 (2010).

${ }^{8}$ G. Isella et al., Solid State Electron. 48, 1317 (2004).

${ }^{9}$ J. Osmond et al., Appl. Phys. Lett. 94, 201106 (2009).

${ }^{10}$ Y. Ishikawa et al., J. Appl. Phys., 98, 013501 (2005).

${ }^{11}$ J. L. Shay, Phys. Rev. B, 2, 803 (1970).

${ }^{12} \mathrm{H}$. Shen et al., Appl. Phys. Lett. 48, 653 (1986).

${ }^{13}$ D. J. Paul, Phys. Rev. B 77, 155323 (2008).

${ }^{14}$ N. Ozguven and P. C. McIntyre, Appl. Phys. Lett., 92181907 (2008).

${ }^{15}$ M. Bonfanti et al., Phys. Rev. B 78, 041407 (2008).

${ }^{16}$ M. De Seta et al., Appl. Phys. Lett. 95, 051918 (2009).

${ }^{17}$ J.-M. Baribeau, J. Vac. Sci. Technol. B 161568 (1998).

${ }^{18}$ X-C. Liu and D. R. Leadley, J. Phys. D: Appl. Phys. 4350530 (2010). 